\title{
Synthesis of nanocomposite 2-methyl-4-chlorophenoxyacetic acid with layered double hydroxide : physicochemical characterization and controlled release properties
}

\begin{abstract}
A new organic-inorganic hybrid nanocomposite Zn-Al-MCPA-layered double hydroxide (ZAM) was prepared by intercalation of 2-methyl-4-chlorophenoxyacetic acid (MCPA) into $\mathrm{Zn}$-Al-layered double hydroxide (ZAL) at various concentration of MCPA ranging from 0.1 to $0.7 \mathrm{M}$. The $\mathrm{pH}$ of the synthesis was kept constant at 7.5. Well-ordered hybrid nanocomposite was obtained with 0.4 M MCPA with an expansion of basal spacing from 8.9 $\AA$ in the ZAL to $19.7 \AA$ in the resulting nanocomposite. The FTIR spectra of the nanocomposite show resemblance peaks of the MCPA and Zn-Al-layered double hydroxide indicating the inclusion of MCPA into the layered double hydroxide. The average particle size of ZAL and ZAM in this study was 115 and $128 \mathrm{~nm}$, respectively. Percentage loading of MCPA was found to be $45.0 \%(\mathrm{w} / \mathrm{w})$, calculated based on the percentage of carbon in the sample. The release of MCPA into various aqueous solution was found to be dependent to the anion in the aqueous solution in the order of phosphate $>$ sulfate $>$ chloride with the percentage release of 80,44 , and $8 \%$, respectively. This study shows that $\mathrm{Zn}-\mathrm{Al}$-layered double hydroxide can be used as a host carrier for herbicide, MCPA, with controlled release capability.
\end{abstract}

Keyword: 2-Methyl-4-chlorophenoxyacetic acid; Herbicides; Hybrid nanocomposite; Layered double hydroxide. 